\title{
2 A role for CD4 helper cells in HIV control and progression
}

3

13 It remains unclear why HIV persists in most untreated individuals, and why a small

14 minority of individuals can control the virus, either spontaneously or after an early 15 treatment. The present work motivated by the striking differences in the functional

16 avidity of CD4 T cells discovered between patient cohorts in a recent study [1] offers

17 an experimentally-testable mathematical model that explains the diverse outcome of

18 infection. The model predicts an arms race between viral dissemination and the

19 proliferation of HIV-specific CD4 helper cells leading to one of two states: a low-

20 viremia state or a high-viremia state. Helper CD4 cells with a higher avidity favor

21 virus control. The parameter segregating spontaneous and post-treatment

22 controllers is the infectivity asymmetry between activated and resting CD4 T cells.

23 The predictions are found to be consistent with the data from [1] and with data on

24 the avidity CD8 $\mathrm{T}$ cells [2]. I also analyze the alternative explanation of $\mathrm{T}$ cell 25 exhaustion previously proposed to explain the diverse patient cohorts and 26 demonstrate that it does not explain these and some other experimental data. 
Submitted Manuscript: Confidential

\section{Importance}

32

Why HIV persists in most untreated individuals, and why a minority can control the virus, either spontaneously or after short treatment, remains unknown. The present study offers a mathematical model of the immune response that explains the differences between the patient cohorts as a result of the arms race between viral dissemination and the proliferation of HIV-specific CD4 T cells. It offers testable predictions and personalized adjustment of early ART to a patient.

\section{Introduction}

Untreated HIV infection causes a gradual loss of CD4 T cells that eventually causes AIDS. Among possible reasons of the slow depletion are impaired homeostasis [3] and a viral adaptation to the host [4]. However, a small fraction of individuals is capable of controlling HIV replication even in the absence of antiretroviral treatment (ART) $[5,6]$. These "controllers" exhibit low viremia undetectable by standard assays, far below their levels in patients receiving ART [7-9]. Importantly, HIV controllers very slowly progress to AIDS if at all [7], confirming the link between a low viral load and the delay of AIDS symptoms $[4,10]$.

Substantial evidence demonstrates that controllers suppress HIV replication due to an active immune response [11]. CD8 T cells rapidly kill infected CD4 T cells through cytotoxic mechanisms involving perforin and Granzyme B $[12,13]$. The signs and markers of immune activation are much more pronounced in HIV controllers than in ART patients, including immunoproliferation rate [14], markers of $\mathrm{T}$ cell activation [15], and the increased secretion of cytokines IFN- $\gamma$ and MIP-1 $\beta$ after stimulation with polyclonal antibodies [16]. While ART patients with undetectable

57 viral loads show a gradual fading of the cellular immune responses [17], HIV 58 controllers maintain polyfunctional effector memory $\mathrm{T}$ cells that can secrete multiple 59 cytokines [18-20]. 
Submitted Manuscript: Confidential

61 among individuals remains the strongest genetic determinant of HIV progression [21-

62 24]. An experimental study demonstrated that HLA-B57, the allele the most 63 associated with HIV control, has unusually broad cross-recognition of antigenic

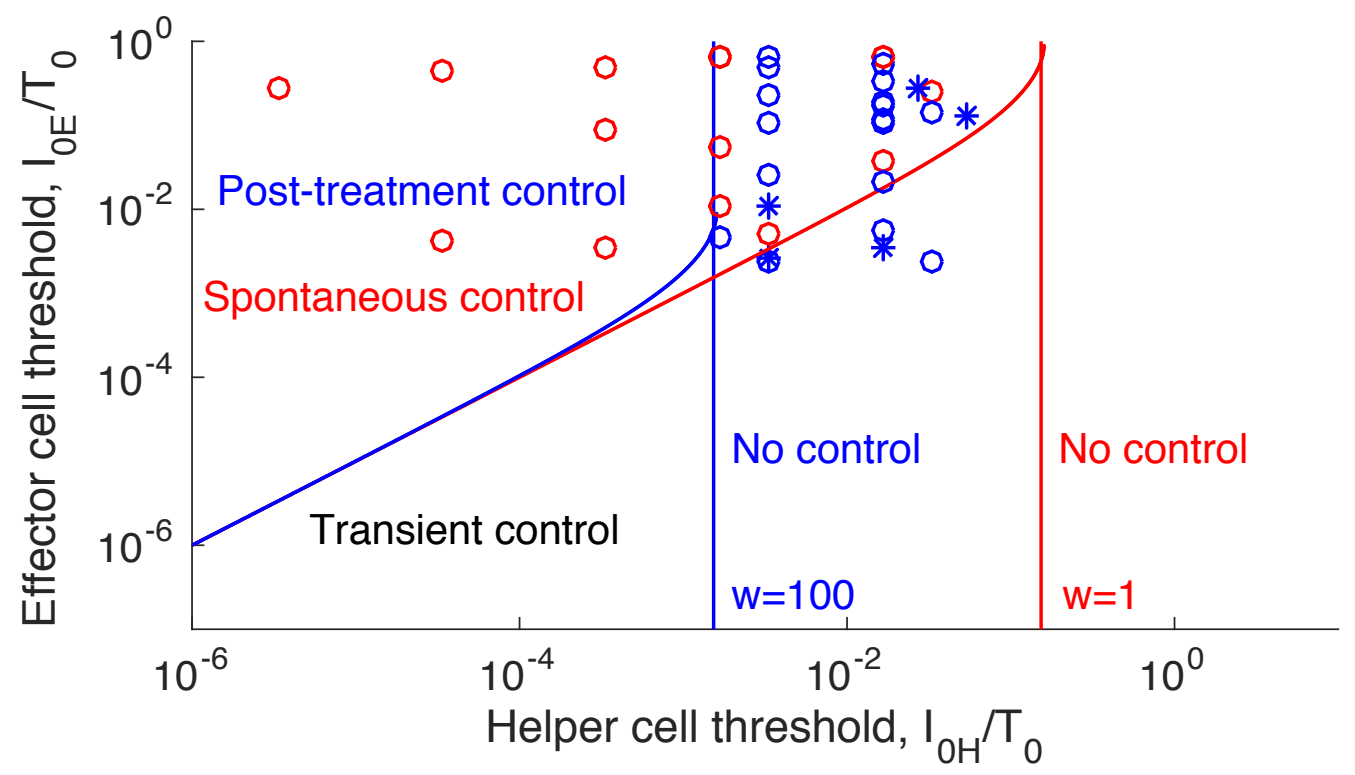

Fig. 1. The difference in CD4 cell avidity between controllers and progressors based on [1]. $\mathrm{X}$-axis: Rescaled inverse functional avidity of CD4 T cells, denoted $I_{H 0}$, measured in three different cohorts of patients [1]. Y-axis: simulated points of the inverse avidity of CD8 T cells, denoted $I_{E 0}$, distributed in a way similar to data from patients in another study [2]. Two scaling parameters are used for the two avidities, one for each axis. Red symbols (o) correspond to spontaneous controllers, and blue symbols $\left(0,{ }^{*}\right)$ correspond to untreated patients with high viremia and patients on long-term ART, respectively. Blue and red lines show the phase diagrams predicted in this work at two different values of infectivity ratio between activated and resting CD4 T cells, $w=$ 100 and $w=1$. The other parameters are given in Table 1.

64 variants of virus [25]. A number of studies compared between patient cohorts the 65 functional avidity, a parameter quantitating the ability of T cells to respond to antigen. 66 In some studies, CD8 T-cell responses in HIV controllers were associated with a high 67 avidity of CD8 T cells [26-29], whereas the other studies indicated a similar avidity in 68 progressors and controllers [30-34]. A recent work concluded this debate 
Submitted Manuscript: Confidential

69 demonstrating that that the range of the functional avidity of HIV-specific CD8 T cells

70 does not differ much between a progressive and non-progressive infection [2].

71 A striking feature setting the two cohorts apart is an active CD4 T cell response

72 with IL-2 secretion capacity and proliferative responses observed in controllers but

73 not in progressors [16]. In a recent study, the functional avidity of Gag293-specific

74 CD4 T cells was compared between spontaneous controllers, high viremia patients,

75 and patients who underwent highly active ART [1]. The avidity was evaluated by IFN-

$76 \gamma$ ELISPOT assay in the presence of decreasing peptide concentrations with serial

77 dilutions from $4 \times 10^{-6} \mathrm{M}$ to $10^{-11} \mathrm{M}$. For each peptide dilution, IFN- $\gamma$ production

78 was expressed as the number of spot-forming cells per million cells. The functional

79 avidity was defined as the last peptide dilution that gave a positive IFN- $\gamma$ response 2-

80 fold above the background level or higher. HIV controllers contained memory CD4+

81 T cells that differentiated upon antigen stimulation into effector CD4+ T cells with a

82 generally higher functional avidity for an immunodominant Gag epitope than in

83 progressors, consistent with a higher binding affinity of TCR to peptide/MHC complex

84 [1]. Although the avidity in all cohorts of patients was broadly distributed, the 85 distribution for controllers was narrower than for progressors and centered at much

86 higher values (Fig. 1). The explanation for these experimental results is absent.

87 Due to complex dynamics of the host-virus system, mathematical modeling 88 has been employed to describe various aspects of HIV infection [35-44], including the 89 existence of progression and control [45] and the treatment with monoclonal 90 antibodies [46]. The relative roles of the fitness cost of antigen escape from CD8 T cell 91 response and the breadth of recognition have also been addressed [47]. However, the 92 striking difference observed in the distribution of the functional avidity of helper CD4

93 T cells between controllers and progressors, in the absence of such difference for CD8

94 T cells, remains unexplained $[1,2]$. The observation is especially interesting given

95 that genetic markers of CD8 T cell receptors (HLA subtype) remain the strongest 96 predictor of HIV control [21-24].

97 The present work offers a model that explains these findings and the 98 difference in the virus dynamics between controllers and progressors. The starting 99 point is the observation that the avidity of effector CD8 T cells, in terms of the average 
Submitted Manuscript: Confidential

100 antigen concentration in the body, is usually much lower than that of effector CD4 T

101 cells, even though both of them span several orders of magnitude. Indeed, effector

102 CD8 T cell proliferates only on a direct contact with an infected cell expressing MHC-

103 I-peptide complex, while an effector CD4 T cell reacts to extra-cellular soluble antigen

104 presented by specialized MHC-II cells and hence can sense antigen far away from its

105 cellular source [48]. Next, HIV and a few other viruses, such as LCMV [49], have the

106 ability to infect and deplete specifically CD4 cells, either by direct cytotoxicity or

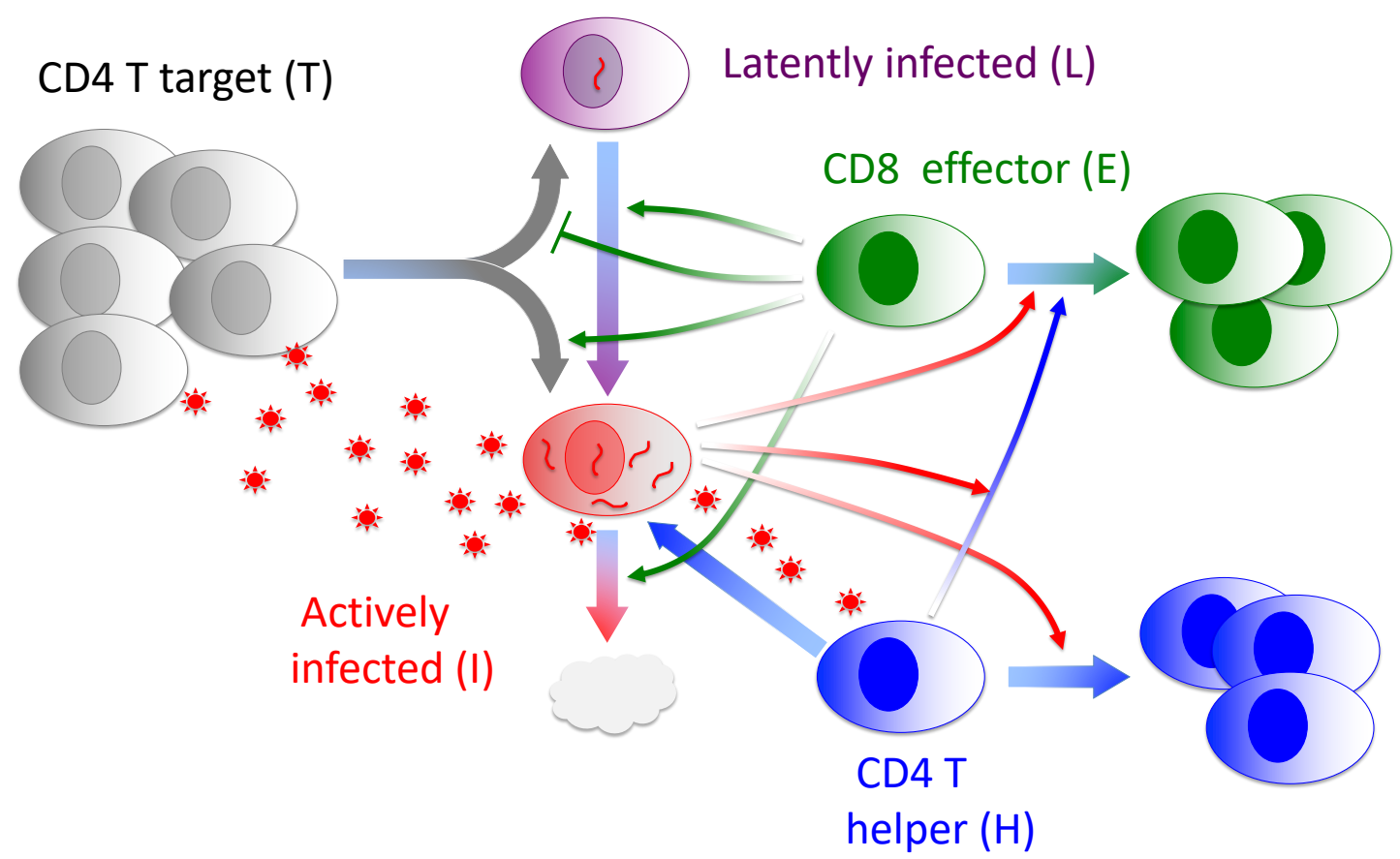

Fig. 2. The model of the immune response and virus dynamics explaining the multiple outcomes of HIV infection. Broad arrows show cell flow and proliferation, thin arrows show control signals discussed in the main text. Little red stars are free virus. Model equations are given in Methods.

107 through killer cells. Taken together, these two observations appear to suggest that 108 the survival strategy of HIV may be to suppress the CD4 T cell response and thus make 109 the immune system much less sensitive to the presence of the virus. Thirdly, it is well 110 known that HIV infects activated CD4 T cells much faster than resting cells [44, 50].

111 The reason is a much higher concentration of nucleotides in activated cells [51].

112 Based on these facts, I propose a model, which predicts the infection outcome based 113 on the knowledge of patient's parameters and explains the difference in CD4 T cells 
Submitted Manuscript: Confidential

114 avidity between patient cohorts. The model is compared to data on T-cell avidity from

115 two recent studies [1], [2]. In Discussion, we discuss the alternative explanation of the

116 dual outcome of HIV infection based on $\mathrm{T}$ cell exhaustion and its connection to

117 experimental data.

118

119

120 Results

121

122 Model. The model comprises five cell compartments, as follows (Fig. 2): uninfected

123 but susceptible target cells of CD4 T CCR5 phenotype, which number is denoted $T$;

124 actively infected cells, $I$; latently-infected cells, $L$; virus-specific helper CD4 T cells,

$125 H$; and cytotoxic effector CD8 T cells, $E$. The biological processes included in the

126 model are, as follows.

127 Target CD4 T cells are replenished, are infected with the virus, and have a finite

128 life span. Actively infected cells are expanded due to new infections and reactivation

129 from latently-infected cells and die due to the virus-induced death and killing by

130 cytotoxic effector CD8 T cells. Latently-infected cells are produced upon infection of

131 target cells and can be reactivated, with the rates of both processes controlled by the

132 number of effector CD8 T cells secreting cytokines activating the integrated HIV

133 provirus [52]. HIV-specific CD4 T helper cells proliferate in response to antigen, with

134 a characteristic threshold (inverse avidity) denoted $I_{H 0}$. These cells are infected at a

135 rate higher than non-specific target cells, by a factor denoted $w, w>1$. The biological

136 justification is that virus-specific cells are activated by antigen, and CD4 T cell

137 infectivity increases with cell activation [44, 50]. CD4 T helper cells also have a finite

138 life span. Finally, HIV-specific CD8 killer cells can proliferate by one of two alternative

139 signaling pathways: either due to binding of TCR to MHC-I-peptide complex

140 expressed by infected cells, with inverse avidity $I_{E 0}$, or due to stimulation of their Il-

1412 receptor by IL-2 secreted by the helper cells. The model equations are given below

142 in Methods. The model parameters including their default values used for simulation

143 are listed in Table 1. 
Submitted Manuscript: Confidential

144

145

146

147

148

149

150

151

152

153

154

155

156

157

158

For the reasons detailed in the Discussion section, the model does not include the exhaustion of CD8 T cells previously proposed to explain the different outcome in controllers and progressors [45]. In Discussion section, we rule out this alternative explanation based on data in Fig. 1 and additional data.

Here I assume that the exhaustion in a chronic infection, which accumulates rather gradually in chronic infection [53], is a consequence of virus persistence rather than its cause, and that its effect on virus dynamics during the first two or three weeks of systemic infection is negligible.

Table 1. Model parameters and their default values in simulation of a representative progressors and controllers. The normal CD4 count, $T_{0}$, and the maximal allowed values of $H, E$ are set to 1 and used as units for the respective variables: $T, I, L$, and $H, E$. The general ranges of parameters are taken from $[43,44]$. The specific (default) values of parameters are adjusted to fit data in Fig. 1 and to obtain oscillation-free dynamics in Figs. 3 and 4, and to match the representative levels of various cell types (see experimental references in [42]).

\begin{tabular}{|c|l|c|c|c|}
\hline Notation & Parameter name & Unit & Default & Range \\
\hline $\boldsymbol{I}_{\mathbf{0 H}}$ & 1/avidity helper cells & $\boldsymbol{T}_{\mathbf{0}}$ & $9 \cdot 10^{-6}$ & $10^{-6}-1$ \\
\hline $\boldsymbol{I}_{\mathbf{0} \boldsymbol{E}}$ & 1/avidity killer cells & $\boldsymbol{T}_{\mathbf{0}}$ & 0.03 & $10^{-6}-1$ \\
\hline $\boldsymbol{W}$ & H to T infectivity ratio & 1 & 10 & $1-100$ \\
\hline $\boldsymbol{C}$ & Max. proliferation rate & $1 /$ day & 2.0 & $1.5-3$ \\
\hline $\boldsymbol{d}_{\boldsymbol{I}}$ & Infected cell death rate & $1 /$ day & 1.0 & $0.7-1.2$ \\
\hline $\boldsymbol{d}_{\boldsymbol{T}}$ & Target cell death rate & $1 /$ day & 0.3 & $0.1-0.5$ \\
\hline $\boldsymbol{d}_{\boldsymbol{H}}$ & Helper cell death rate & $1 /$ day & 0.15 & $0.07-0.3$ \\
\hline $\boldsymbol{d}_{\boldsymbol{E}}$ & Killer cell death rate & $1 /$ day & 0.15 & $0.07-0.3$ \\
\hline $\boldsymbol{r}_{\boldsymbol{m a x}}$ & Max. latency activation rate & $1 /$ day & 0.2 & \\
\hline $\boldsymbol{r}_{\mathbf{0}}$ & Min. latency activation rate & $1 /$ day & 0.001 & \\
\hline $\boldsymbol{p}_{\boldsymbol{L} \mathbf{0}}$ & Max. latency probability & 1 & 0.4 & \\
\hline $\boldsymbol{R}_{\mathbf{0}}$ & Basic reproduction number & 1 & 8 & $4-12$ \\
\hline $\boldsymbol{\varepsilon}$ & Drug efficacy & 1 & 0.97 & \\
\hline $\boldsymbol{I}_{\boldsymbol{i n}}$ & Initial actively infected cell \# & $\boldsymbol{T}_{\mathbf{0}}$ & $10^{-11}$ & $10^{-11}-10^{-9}$ \\
\hline $\boldsymbol{L}_{\boldsymbol{i n}}$ & Initial latent cell \# & $\boldsymbol{T}_{\mathbf{0}}$ & $10^{-11}$ & $10^{-11}-10^{-9}$ \\
\hline $\boldsymbol{E}_{\boldsymbol{i n}}$ & Initial killer cell \# & 1 & $10^{-5}$ & $10^{-7}-10^{-4}$ \\
\hline $\boldsymbol{H}_{\boldsymbol{i n}}$ & Initial helper cell \# & 1 & $10^{-5}$ & $10^{-7}-10^{-4}$ \\
\hline $\boldsymbol{T}_{\mathbf{0}}$ & Normal target cell \# & 1 & 1 & \\
\hline $\boldsymbol{H}_{\mathbf{0}}$ & Acting \# of helper cells & 1 & $10^{-2}$ & \\
\hline $\boldsymbol{E}_{\mathbf{0}}$ & Acting \# of killer cells & 1 & $10^{-2}$ & \\
\hline & & & & \\
\hline
\end{tabular}


bioRxiv preprint doi: https://doi.org/10.1101/2021.12.20.473599; this version posted December 21, 2021. The copyright holder for this preprint (which was not certified by peer review) is the author/funder. All rights reserved. No reuse allowed without permission.

\section{Submitted Manuscript: Confidential}

Two possible steady states. Analysis presented in S1 Text online shows that

161 the system has two possible steady states (fixed points) at which the size of each cell

162 compartment does not change in time because its proliferation is exactly 163 compensated by the cell death, as follows:

164 I. The helper-dependent steady state with a very low virus load observed in a 165 spontaneous or post-treatment controller (S1 Text, Eq. 9). An example of simulation resulting in spontaneous control is presented in Fig. 3A, with the stability region is
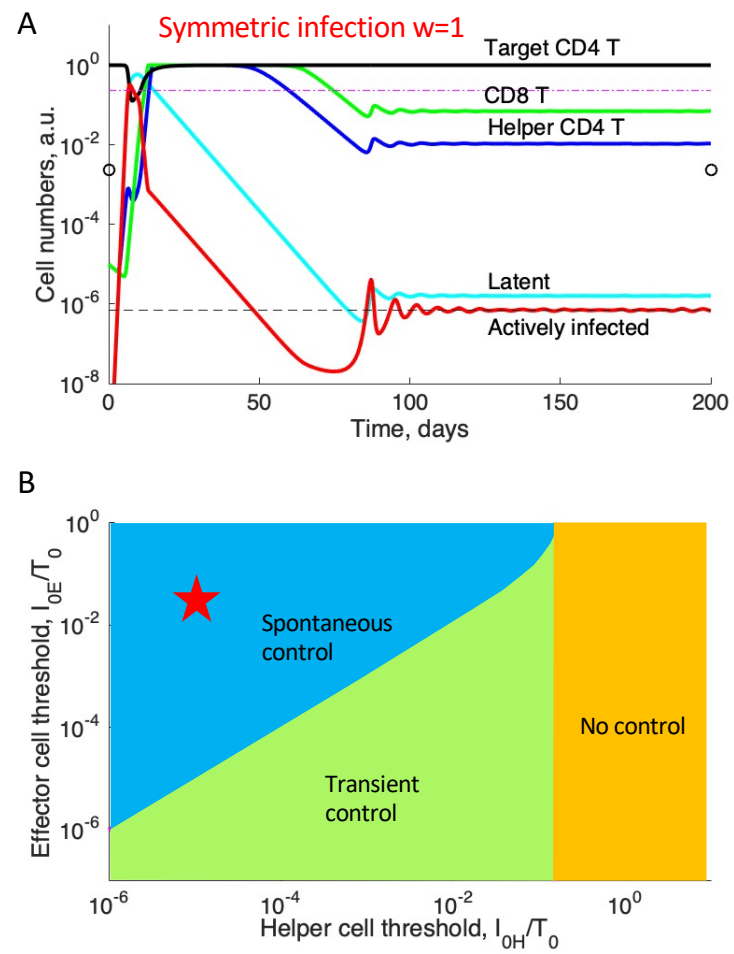

Figure 3. The model predicts the conditions of the spontaneous virus control. Infectivity of virus-specific and non-specific cells is assumed to be the same, $w=1$. (A) Spontaneous establishment of a low-viremia helper-dependent state. Y-axis: Numbers of cell compartments in relative units to their maximal values set at 1 . X-axis: Time of the systemic phase of infection. (B) Phase diagram predicting the four outcomes (S1 Text, Eqs. 15 and 17). X-axis: The helper CD4 T cell threshold in antigen (inverse avidity), $I_{0 H}$, in units of the normal CD4 T cell count, $T_{0}$. Y-axis: The same for killer CD8 T cells. The asterisk shows 1 /avidities used in (A), $I_{0 E}=0.03, I_{0 H}=9 \cdot 10^{-6}$. Notation in (A): Black dashed line is the helper-dependent steady state $I_{1}$ (Eq. 9 in S1 Text). Two little circles show the helper-free steady state $I_{2}$ (Eq. 10 in S1 Text). Magenta dash-dotted line is the limit of helper cell expansion, $I^{*}$, Eq. 11. (A, B) Fixed parameter values are given in Table 1. Relevant parameters in (B): $R_{0}=8, \frac{c}{d_{I}}=2, \frac{c}{d_{E}}=13.3, \frac{c-d_{H}}{d_{I}}=1.85, T_{0} /\left[R_{0} d_{I} \int I(t) d t\right]=0.11$. The last parameter is calculated from simulation and depends on the other parameters. The specific (default) values of parameters are adjusted to fit data in Fig. 1 and to obtain oscillation-free dynamics and representative levels of various cell types (see experimental references in [42]). 
Submitted Manuscript: Confidential

167 shown in the phase diagram (Fig. 3B, blue region). If the avidity of helper cells is 168 sufficiently low, this state becomes unstable (Fig 3B, green) or disappears altogether 169 (Fig 3B, yellow). The exact conditions of its existence and stability are given in S1 Text 170 (Eqs. 15 and 17).

171 II. The helper-independent steady state with a high virus load observed in a 172 progressor or potential post-treatment controller in the absence of treatment (Eq. 173 10). An example of simulation is shown in Fig. 4A.

174 Post-treatment control. Simulation demonstrates that, in the imaginary 175 organism with an unlimited number of T cells, only one of these two states can exist 176 indefinitely, whichever has a lower virus load. In a real organism containing $\sim 10^{11} \mathrm{~T}$ 177 cells, the two states can co-exist. The limited size leads to effective bi-stability, as

A

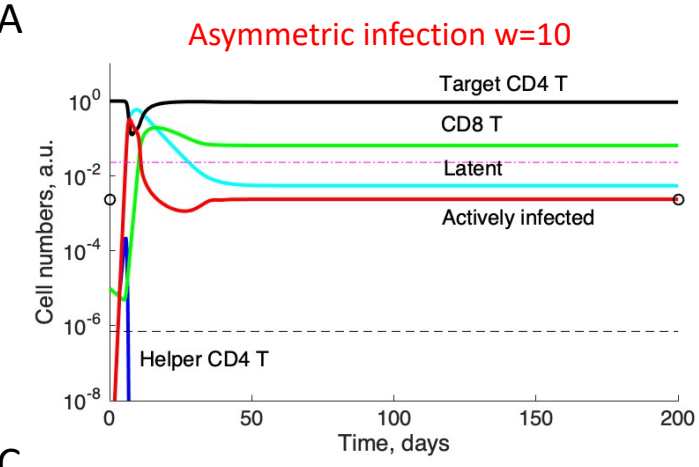

C

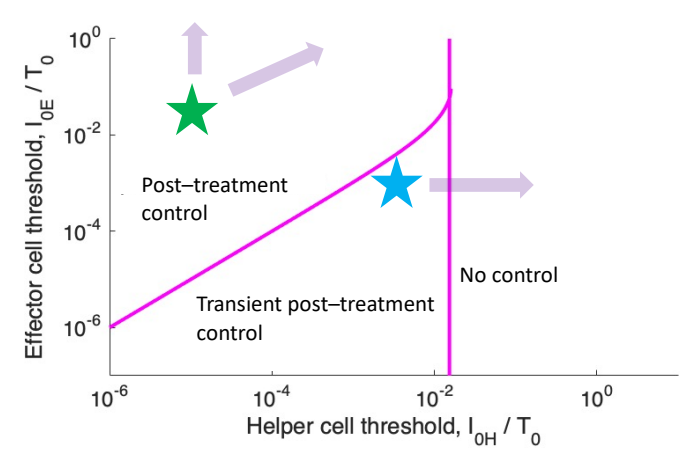

B

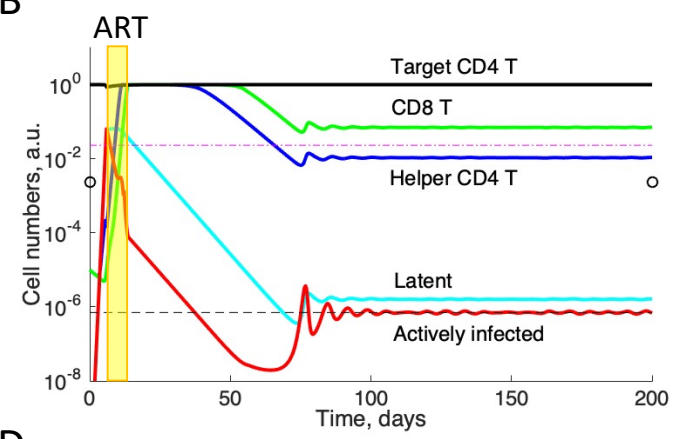

D

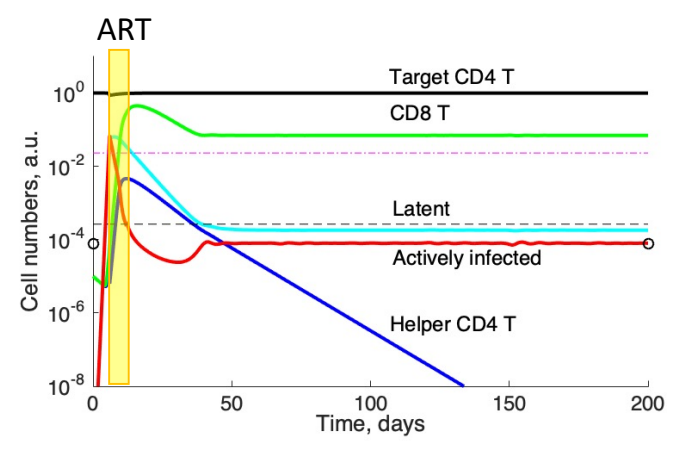

Figure 4. The model predicts the conditions of post-treatment control and transient control. Infectivity of virus-specific (and hence activated) CD4 T cells is assumed to be 10 times higher than for non-specific (and hence resting) CD4 T cells, $w=10$. (A) Early infection and killing of CD4 T cells leads to a high-viremia helper-free state (S1 Text, Eqs. 18, 19). (B) ART between days 6 and 12 with the efficacy of $97 \%$ leads to the rescue of helper cells and establishment of a low-viremia state. (D) If avidity of helper cells is lower than the avidity of killer cells, helper cells decay gradually and control is only transient. (A, B, D) Notation as in Fig. 3A. (C) Phase diagram predicting the three outcomes of infection (Eqs. 15, 17). The asterisks show parameters used in simulation: $(\mathrm{A}, \mathrm{B}) I_{0 E}=$ $0.03, I_{0 H}=9 \cdot 10^{-6}$, (D) $I_{0 E}=0.001, I_{0 H}=0.003$. Relevant parameters in (D) are $w=10, T_{0} /$ $\left[R_{0} d_{I} \int I(t) d t\right]=0.2$ and the other parameters are as in Fig. 3. 
Submitted Manuscript: Confidential

178 follows: if the infectivity ratio of active-to-resting helper cells, $w$, is sufficiently high, 179 helper cells can be completely depleted during the acute phase of infection before 180 they can reach the steady state (S1 Text, Eq. 19). Even though the helper-dependent 181 state would be stable once reached, helper cells can never make it. As a result, the 182 high-viremia state is established forever (Fig. 4A).

183 Simulation demonstrates that the helper cell-dependent, low-viremia state 184 can be recovered by an early anti-retroviral therapy (ART) started at day 6 or 7 of 185 systemic infection (that follows the mucosal phase). Blocking infection of helper cells 186 by ART allows them to expand until they reach the steady state and take control of 187 killer cells (Fig. 4B). Because their activation threshold is very low compared to that 188 of killer CD8 cells, the steady state viremia is very low, relatively speaking, which 189 effect explains the effect of post-treatment control.

190 If the avidity of helper cells is sufficiently small, the post-treatment state will 191 be only transiently stable, helper cells will decay gradually, and the system will 192 eventually slide into the high-viremia state (Fig. 4D).

193 Phase diagram of patient cohorts. Combining all these cases (Eqs. 15, 17, 194 and 19 in S1 Text, and Fig. 3B and 4C) results in a phase diagram in coordinates $195\left(I_{0 H}, I_{0 E}\right)$, with four distinct regions corresponding to four different cohorts, as 196 follows:

i) Spontaneous control. A stable helper response and low viremia in the form 198 of oscillations emerge spontaneously (Fig 3A). No treatment is required.

ii) Post-treatment control. Untreated patients lose helper cells rapidly due to HIV infection (Fig. 4A), but an early antiretroviral treatment rescues helper cells and 201 establishes a low-viremia state (Fig. 4B).

iii) Transient (unstable) post-treatment control. Helper cells can reach high 203 levels but then decay gradually (Fig. 4D).

204 iv) Progressors. The helper-free state with a high virus load is the only possible 205 state, and its dynamics is similar to that in Fig. 4A.

206 The phase diagram in Fig. 3B and 4C is consistent with the clinical observation 207 that controllers harbor helper CD4 T cells with a higher avidity than progressors [1]. 208 It also demonstrates the importance of an asymmetric infection rate [44, 50]. 
Submitted Manuscript: Confidential

These results also demonstrate that HIV takes advantage of latency hard210 wired into its genetic circuitry [52] to avoid full clearance in controllers by frequent 211 reactivation from latent cells (Fig. 3A and 4B). Another evolutionary role for latency 212 is to improve the chances of viral survival upon mucosal entry [42]. The two 213 evolutionary roles can be combined stating that latency is the method used by HIV to 214 survive population bottlenecks, similar to spores of monocellular organisms [42].

215 In the phase diagrams (Figs. 3B and 4C), spontaneous controllers differ from 216 post-treatment controllers by an unusually low infectivity asymmetry, $w$. Because $w$ 217 is typically quite high [43,44], post-treatment control (Fig. 4C) is predicted to be 218 much more likely than the spontaneous control (Fig. 3B), which agrees with 219 observations (10-15\% and $0.5 \%$ of patients, respectively). This prediction is testable, 220 both in vivo and ex vivo.

221 An interesting feature is the prediction of a transient controller state, 222 characterized by the slow decay of helper cells and gradual switch to the direct 223 antigen activation (Fig. 3E). This explains, at least, qualitatively, why control is often

224 transient. In principle, control in this state could be prolonged by periodic short-term 225 treatment resetting helper cells to a high level. However, the safety of such a 226 hypothetical protocol, in view of the danger of drug-resistance mutants, would 227 require careful testing.

In addition to predicting the outcome of an individual infection, the model 229 predicts the best timing for ART. Simulation (Fig 4B) demonstrates that ART must 230 start between days 6 and 8 of the systemic (post-mucosal) phase of infection. The 231 exact time window depends on patient's parameters. The rather strict timing 232 requirement raises the possibility that the observed post-treatment controllers may 233 be only a small fraction of their potential number. In principle, the application of this 234 model could enable physicians to improve the timing of early ART and expand the 235 number of post-treatment controllers. Whether this idea is practical, remains to be 236 seen. 
Submitted Manuscript: Confidential

240 low value, $w=1$. Note that the region of virus control is smaller in the first case (blue

241 lines). To test this diagram experimentally, one has to plot on top of it the pairs of

242 avidity values obtained for real patients. Data for the avidity of both T cell types

243 measured in the same study are not available. To partly circumvent this problem, the

244 actual data set on CD4 cell 1/avidity, $I_{H 0}$, obtained for spontaneous controllers,

245 progressors, and patients on ART in [1] is combined with a simulated set for $I_{E 0}$

246 resembling experimental ldata for CD8 T cells obtained in [2] (Fig. 1). Based on the

247 result of [2], the same distribution function for the CD8 T cell avidity for all three

248 patient cohorts is assumed. Because both avidities are defined within a constant

249 scaling factor, the two scaling factors are adjusted to fit the data in the diagram; the

250 same two scaling factors are used for all cohorts. Thus, the model explains the

251 observation that progressors have a relatively low CD4 T cell avidity (high $I_{H 0}$ ).

252 Spontaneous control is predicted to be a result of two rare events: unusually

253 high CD4 T cell avidity and an unusually low value of $w$. This prediction explains why

254 it spontaneous control so rare (0.5\%). In contrast, post-treatment control requires

255 only a high CD4 T cell avidity but asymmetry can by typically high.

256 Robustness to parameter values. So far, the focus was on three parameters

$257\left(I_{0 H}, I_{0 E}\right.$, and $\left.w\right)$ keeping the other model parameters in Table 1 fixed. The sensitivity

258 of the phase diagram to the other parameters is tested with plots made in another

259 pair of axes, $\left(I_{H 0}, w\right)$, while keeping 1 /avidity of CD8 T cells, $I_{0 E}$, fixed (S1 Fig). The

260 border between progressors and transient controllers is rather robust to all

261 parameters. In contrast, the boundary separating spontaneous control and post-

262 treatment control is sensitive to most parameters. Parameters that most significantly

263 alter the external boundary of post-treatment control are $d_{H}$ and $d_{E}$. A particular

264 form of the control functions, $\alpha(x), \beta(y)$ in Eq. 1, is tested not to be very important

265 for results, as long as $\alpha(x)$ changes between $x$ and 1 at small and large $x$, and $\beta(y)$

266 changes between 1 and 0 at small $y$ and $y=1$. The reason for this robustness is that

267 the system has very fast dynamics and spends most of its time at the values of $x$ either

268 much larger or much smaller than 1 , and at $y$ much smaller than 1. 
Submitted Manuscript: Confidential

270

271

272

273

274

275

276

277

278

279

280

281

282

283

284

285

286

287

288

289

290

291

292

293

294

295

296

297 298

\section{Discussion}

Thus, the model explains why controllers have higher avidity of CD4 T cells than progressors, and explains why post-treatment control is more frequent that spontaneous control. The outcome of HIV infection can be understood from the two signals of CD8 $\mathrm{T}$ cell activation, one through T-cell receptor binding to MCH-I-peptide complex of infected cells, and another through IL-2 secretion by virus-specific CD4 T helper cells. The model predicts two possible steady states, one state with a high level of helper cells and a low virus load determined by the maintenance of helper cell population by MHC-II dependent activation, and the other state without helper cells, where the high virus load is self-tuned to maintain the CD8 T cell population by MHCI dependent activation. The first state is characterized by a very slow progression to AIDS.

Which state is stable, depends, mostly, on three parameters: the avidities of helper cells and CD8 T cells, and the asymmetry of infectivity between antigenactivated and non-virus-specific CD4 T cells. If antigen-specific helper CD4 cells are much more infectable than non-HIV specific CD4 cells $(w>2-3)$, which is expected to be the case in most patients, helper cells are completely depleted by virus before they even reach the steady state. As a result, the system arrives at a helperfree state with a high virus load. If, however, a short therapy is administered early, roughly two weeks after infection, infection is transiently suppressed, helper cells are rescued, and the system arrives at the low-viremia state.

Spontaneous control is predicted as a result of two unlikely events: unusually high CD4 T cell avidity and an unusually low value of $w$, in the tail of its distribution. This prediction explains why it is so rare (0.5\%). In contrast, post-treatment control requires only a high CD4 T cell avidity but asymmetry can by typically high, which explains why post-treatment control is more frequent that spontaneous control. Thus, not only different outcomes are explained, but also their probabilities and the data separation. 
Submitted Manuscript: Confidential

The present model assumed that all CD4 T cells are killed by productive infection. In fact, $95 \%$ of T cells die after an abortive infection [54]. There is no need, however to introduce this parameter and repeat simulations again. It suffices to rescale $T_{0} \rightarrow 0.05 T_{0}$. Indeed, effectively, only $5 \%$ of permissive cells become host

303 cells. That means that all the characteristic values of the infected cell number, such as

$304 I_{0 H}$ and $I_{0 E}$, must be rescaled by the scaling factor of 0.05 . Therefore, the indirect 305 death does not change the shape of the phase diagram, as long as $I_{0 H}$ and $I_{0 E}$ are 306 scaled in units of $0.05 T_{0}$ (Figs. $2 \mathrm{~B}$ and $3 \mathrm{C}$ ). One must use the rescaling factor when 307 comparing them with real data, where $I_{0 H}$ and $I_{0 E}$ are measured in units of cell $/ \mathrm{ml}^{3}$.

308 The model predicts a controller state with either flat viremia or periodic oscillations around the steady state value (Fig. 2A or 3B). In fact, viral blips are observed in many patients due to the activation of virus from latent cells modeled

311 earlier in ART patients [55]. This stochastic process is better described by a Gillespie 312 simulation than deterministic equations [42].

313 The exact timing of early ART is of essence. The best timing can be predicted

314 from the present model informed by the avidities of both $\mathrm{T}$ cell types based on the

315 HLA subtypes of the patient. Thus, the present study offers not only a mechanistic 316 method to understand and predict the outcome of HIV infection in an individual, but 317 also a hope to expand the number of post-treatment controllers in the future therapy.

318 Variation of HLA CD8 T cell subtypes remains the strongest genetic 319 determinant of HIV progression [21-24]. Yet, the present model does not predict any 320 interesting dependence on the properties of CD8 T cells. This is because our simple 321 model of immune dynamics (Fig. 2) does not take into account that the virus can 322 mutate in epitopes. In real patients, HIV undergoes up to 20 or 30 primary escape 323 mutations in multiple epitopes, with a widely variable fitness cost [56-60]. The order 324 of these mutations is predicted to be determined by the balance between the partial 325 loss of immune recognition per mutation and its fitness cost [47]. Escape mutations 326 can occur very early in the acute phase, during the peak of infection. This fact limits 327 the longevity of the otherwise stable low-viremia state. For it to be long-lived, an 328 escape from CD8 T cell response controlled by helper cells has to be slow, which 329 requires either a high mutation cost or a small recognition loss per mutation [47]. 
Submitted Manuscript: Confidential

330 This requirement may explain why specific HLA subtypes of CD8 T cells correlate with 331 control (see [24] and references therein). In keeping with this prediction, an 332 experimental study from MIT has demonstrated that HLA-B57, the allele the most 333 associated with HIV control, has unusually broad cross-recognition, thus confirming 334 the role of antigenic escape in limiting virus control [25].

335 Previously, the exhaustion of CD8 T cells has been proposed to explain the 336 difference between controllers and progressors [45]. Citing the evidence of the CD8 337 T cell anergy in a long-term HIV infection $[53,61]$, the authors of the model postulate 338 that sufficiently high virus loads can disable virus-specific effector CD8 cells in the 339 acute phase of infection. The acute phase of infection, according to [45], is a battle 340 between the viral expansion that disables CD8 T cells and the expansion of CD8 T cells 341 that kill the infected cells. In patients with a low killing CD8 cell ability, in which case 342 the virus peak is tall enough to suppress the immune response, the virus wins the 343 battle resulting into a high-viremia steady-state. The rare patients with an unusually 344 high killing ability reach high levels of CD8 response with no virus replication in the 345 end, which are the controllers. Finally, patients with an intermediate killing potential 346 are the post-treatment controllers that reach a relatively low viremia after early 347 treatment. The model was also used to interpret the results of treatment of 348 experimentally infected animals with broadly neutralizing antibodies [46] and is now 349 being applied to rare chronic cases of SARS CoV-2. While a valuable attempt at 350 explaining dichotomy "control-progression", this model has a number of issues 351 regarding its connection to the actual virology and pathogenesis of HIV, as follows:

352 i. The model [45] postulates instant CD8 T cell exhaustion as a dominant force 353 within the acute infection. In fact, CD8 $\mathrm{T}$ cell anergy, which represents a 354 defense mechanism against immunopathology, is associated with long-term 355 chronic infections, including HIV and some LCMV strains [49, 53, 61 ]. 356 Accumulation of anergic cells in an HIV infection is gradual and progressive [53]. In accordance with this fact, T cell "exhaustion" has been modeled either through a time integral of virus load $[62,63]$ or through impairment/infection of an additional cell compartment [64]. 
Submitted Manuscript: Confidential

ii. Given that the outcome (progressive vs control) is decided within the first weeks of infection [11], and that exhaustion is a gradual progressive process [53], it is unclear how the second can be the cause of the first. More likely, it is the other way around.

iii. Further, the cited model makes no attempt to use of virological details specific for HIV of LCMV, which viruses show long-term persistence and exhaustion markers. It does not explain why influenza virus does not persist, despite comparably high peak virus loads. In particular, the model does not take an advantage of the specific tropism of both HIV and LCMV for helper CD4 T cells.

iv. Although CD4 T cell depletion in progressors results in AIDS, the "exhaustion" model fails to recognize CD4 T helper cells as a critically important part of the immune system and does not even include them into consideration [45]. As already mentioned, the avidity of CD4 cells differ drastically between progressors and controllers $[1,65,66]$, which fact remains unexplained.

v. The model predicts zero replication in spontaneous controllers [45]. In contrast to the prediction, the active replication levels in spontaneous controllers are never zero, even though some of them are below the detection threshold (1000 to 10,000 actively infected cells in the body) [11].

vi. The exhaustion model predicts that CD8 $\mathrm{T}$ cells in controllers have a higher killing capacity than in progressors [45], which, to my knowledge, is not supported by any data. It also predicts a lower viremia peak for controllers than for progressors, which has not been observed either. If anything, controllers have a higher peak. In fact, the controlling HLA subtypes are associated with a broader cross-reactivity [25], which make them less prone to antigenic escape [47].

vii. Although cellular markers of "exhaustion" are known, its molecular mechanisms remain obscure [53], which makes the model difficult to test or falsify .

In conclusion, the present study offers a model of two-signal activation of CD8 $\mathrm{T}$ cells that explains the unusually high avidity of helper cells in controllers and 
Submitted Manuscript: Confidential

391 predicts a testable connection between HIV control, the avidity of CD4 T cells, and the dependence of HIV infectivity on cell activation status. The last prediction merits

393 further experimental investigation.

\section{Methods}

396

397 The time dependence is described by ordinary differential equations for the number

398 of CD4 T cells infectable by virus, $T$, actively infected cells, $I$, latently infected cells, $L$,

399 helper CD4 T cells, $H$, and cytotoxic effector CD8 T cells, $E$, for the model in Fig. 2, have 400 a form

$$
\begin{gathered}
\frac{d T}{d t}=b-p I T-d_{T} T \\
\frac{d I}{d t}=\left[1-p_{L}(E)\right] p I T-d_{I}\left(1+\frac{E}{E_{0}}\right) I+r(E) L \\
\frac{d L}{d t}=p_{L}(E) p I T-r(E) L \\
\frac{d H}{d t}=c H \alpha\left(\frac{I}{I_{0 H}}\right) \beta\left(\frac{H}{H_{m}}\right)-p w I H-d_{H} H \\
r(E)=r_{0}+r_{m a x} \frac{E}{E+E_{0 L}}, p_{L}(E)=p_{L 0} \frac{E_{0 L}}{E+E_{0 L}} \\
\left.\left.\alpha(x) \equiv 1-e^{-x}, \beta(y) \equiv(1-y)^{\frac{1}{3}}, p=0 \text { if } I<\frac{I}{I_{0 H}}\right)\right] \beta\left(\frac{E}{E_{m}}\right)-d_{E} E
\end{gathered}
$$

408 Equation 1 describes the replenishment of target cells (CD4 T+ CCR5+ cells), $T(t)$,

409 with linear rate $b$, their infection with infectivity parameter $p$ and their natural life $410 \operatorname{span} 1 / d_{T}$. Equation 2 describes the expansion and death of actively infected cells,

$411 I(t)$, as well as their reactivation from latently infected cells, $L$. The death rate 412 includes virus-induced death with rate $d_{I}$ and killing by cytotoxic effector CD8 T cells, $413 E(t)$. Equation 3 represents the expansion and reactivation of latently infected cells, $414 L(t)$, where $p_{L}(E)$ is the probability of latency and $r(E)$ is the probability of 415 reactivation depending on the level of cytokines secreted by effector cells, $E$ [42]. 
Submitted Manuscript: Confidential

416

417

418

419

420

421

422

423

424

425

426

427

428

429

430

431

432

433

434

435

436

437

438

439

\section{0}

441

442

443

444

445

446

447

448

Equation 4 states that the dominant subset of CD4 T helper cells recognizing HIV, $H(t)$, proliferates in response to infected cells with maximal rate $c$ but cannot exceed a ceiling, $H_{m}$. In the absence of activation, helper cells retire at rate $d_{H}$. The activation-induced death is included in the net proliferation rate, $c$. Helper cells can be infected at rate $w p$, which is larger than the infection rate of target cells, $T$, by a large factor $w$, as given by $w \gg 1$. The CD4 T cells infectivity can vary by 2-3 orders of magnitude depending on a cell activation status $[44,50]$. The concentration of nucleotides is much higher in S phase of the cell cycle than in G1 phase. HIV protein VPR arrests the host cell in G2 phase to optimize nucleotide concentration and thus expedite the reverse transcription of HIV RNA to proviral DNA [51].

Finally, Equation 5 describes the expansion of effector CD8 cells, $E(t)$, due to two alternative signaling pathways: from infected cells through TCR to MHC-Ipeptide binding, and from helper cells through Il-2 stimulation. For the sake of simplicity, I assume that these signals are additive. As I have checked by simulation, the results are not very sensitive to the linear approximation or the form of control functions $\alpha$ and $\beta$. The ranges of model parameters given in Table 1 are based on [44]. Analysis of these equations is given in S1 Text online.

Parameter choice. The aim is to obtain a representative example of dual outcome and fit data in Fig. 1. The general ranges of parameters are taken from [43, 44]. The specific (default) values of parameters are adjusted to fit data in Fig. 1 and to obtain oscillation-free dynamics in Figs. 3 and 4, and to match the representative levels of various cell types (see experimental references in [42]).

\section{References}

1. Vingert B, Perez-Patrigeon S, Jeannin P, Lambotte O, Boufassa F, Lemaitre F, et al. HIV controller CD4+ T cells respond to minimal amounts of Gag antigen due to high TCR avidity. PLoS Pathog. 2010;6(2):e1000780. Epub 2010/03/03. doi: 10.1371/journal.ppat.1000780. PubMed PMID: 20195518; PubMed Central PMCID: PMCPMC2829066.

2. Vigano S, Bellutti Enders F, Miconnet I, Cellerai C, Savoye AL, Rozot V, et al. Rapid perturbation in viremia levels drives increases in functional avidity of HIVspecific CD8 T cells. PLoS Pathog. 2013;9(7):e1003423. Epub 2013/07/16. doi: 
Submitted Manuscript: Confidential

10.1371/journal.ppat.1003423. PubMed PMID: 23853580; PubMed Central PMCID: PMCPMC3701695.

3. Okoye AA, Picker LJ. CD4(+) T-cell depletion in HIV infection: mechanisms of immunological failure. Immunol Rev. 2013;254(1):54-64. Epub 2013/06/19. doi: 10.1111/imr.12066. PubMed PMID: 23772614; PubMed Central PMCID: PMCPMC3729334.

4. Rouzine IM. An evolutionary model of progression to AIDS. Microorganisms. 2020;8(11). Epub 2020/11/05. doi: 10.3390/microorganisms8111714. PubMed PMID: 33142907; PubMed Central PMCID: PMCPMC7692852.

5. Deeks SG, Walker BD. Human immunodeficiency virus controllers: mechanisms of durable virus control in the absence of antiretroviral therapy. Immunity. 2007;27(3):406-16. Epub 2007/09/26. doi: 10.1016/j.immuni.2007.08.010. PubMed PMID: 17892849.

6. Saez-Cirion A, Pancino G, Sinet M, Venet A, Lambotte O, group AEHCs. HIV controllers: how do they tame the virus? Trends Immunol. 2007;28(12):532-40. Epub 2007/11/06. doi: 10.1016/j.it.2007.09.002. PubMed PMID: 17981085.

7. Lambotte O, Boufassa F, Madec Y, Nguyen A, Goujard C, Meyer L, et al. HIV controllers: a homogeneous group of HIV-1-infected patients with spontaneous control of viral replication. Clin Infect Dis. 2005;41(7):1053-6. Epub 2005/09/06. doi: 10.1086/433188. PubMed PMID: 16142675.

8. Sajadi MM, Heredia A, Le N, Constantine NT, Redfield RR. HIV-1 natural viral suppressors: control of viral replication in the absence of therapy. AIDS. 2007;21(4):517-9. Epub 2007/02/16. doi: 10.1097/QAD.0b013e328013d9eb. PubMed PMID: 17301571.

9. Viard JP, Burgard M, Hubert JB, Aaron L, Rabian C, Pertuiset N, et al. Impact of 5 years of maximally successful highly active antiretroviral therapy on CD4 cell count and HIV-1 DNA level. AIDS. 2004;18(1):45-9. Epub 2004/04/20. doi: 10.1097/00002030-200401020-00005. PubMed PMID: 15090828.

10. Arnaout RA, Lloyd AL, O'Brien TR, Goedert JJ, Leonard JM, Nowak MA. A simple relationship between viral load and survival time in HIV-1 infection. Proc Natl Acad Sci U S A. 1999;96(20):11549-53. Epub 1999/09/29. doi: 10.1073/pnas.96.20.11549. PubMed PMID: 10500214; PubMed Central PMCID: PMCPMC18071.

11. Gonzalo-Gil E, Ikediobi U, Sutton RE. Mechanisms of Virologic Control and Clinical Characteristics of HIV+ Elite/Viremic Controllers. Yale J Biol Med. 2017;90(2):245-59. Epub 2017/06/29. PubMed PMID: 28656011; PubMed Central PMCID: PMCPMC5482301.

12. Migueles SA, Osborne CM, Royce C, Compton AA, Joshi RP, Weeks KA, et al. Lytic granule loading of CD8+ T cells is required for HIV-infected cell elimination associated with immune control. Immunity. 2008;29(6):1009-21. Epub 2008/12/09. doi: 10.1016/j.immuni.2008.10.010. PubMed PMID: 19062316; PubMed Central PMCID: PMCPMC2622434.

13. Saez-Cirion A, Lacabaratz C, Lambotte O, Versmisse P, Urrutia A, Boufassa F, et al. HIV controllers exhibit potent CD8 T cell capacity to suppress HIV infection ex vivo and peculiar cytotoxic T lymphocyte activation phenotype. Proc Natl Acad Sci U S A. 2007;104(16):6776-81. Epub 2007/04/13. doi: 10.1073/pnas.0611244104. PubMed PMID: 17428922; PubMed Central PMCID: PMCPMC1851664. 
Submitted Manuscript: Confidential

495 14. Brenchley JM, Price DA, Schacker TW, Asher TE, Silvestri G, Rao S, et al. 496 Microbial translocation is a cause of systemic immune activation in chronic HIV 497 infection. Nat Med. 2006;12(12):1365-71. Epub 2006/11/23. doi: 10.1038/nm1511. 498 PubMed PMID: 17115046.

499 15. Hunt PW, Brenchley J, Sinclair E, McCune JM, Roland M, Page-Shafer K, et al. 500 Relationship between $\mathrm{T}$ cell activation and CD4+ T cell count in HIV-seropositive 501 individuals with undetectable plasma HIV RNA levels in the absence of therapy. J 502 Infect Dis. 2008;197(1):126-33. Epub 2008/01/04. doi: 10.1086/524143. PubMed 503 PMID: 18171295; PubMed Central PMCID: PMCPMC3466592.

504 16. Potter SJ, Lacabaratz C, Lambotte O, Perez-Patrigeon S, Vingert B, Sinet M, et 505 al. Preserved central memory and activated effector memory CD4+ T-cell subsets in 506 human immunodeficiency virus controllers: an ANRS EP36 study. J Virol.

507

508

509

510

511

512

513

514

515

516

517

518

519

520

521

522

523

524

525

526

527

528

529

530

531

532

533

534

535

536

537

538

539 2007;81(24):13904-15. Epub 2007/10/12. doi: 10.1128/JVI.01401-07. PubMed PMID: 17928341; PubMed Central PMCID: PMCPMC2168869.

17. Pitcher CJ, Quittner C, Peterson DM, Connors M, Koup RA, Maino VC, et al. HIV1-specific CD4+ T cells are detectable in most individuals with active HIV-1 infection, but decline with prolonged viral suppression. Nat Med. 1999;5(5):518-25. Epub 1999/05/06. doi: 10.1038/8400. PubMed PMID: 10229228.

18. Betts MR, Nason MC, West SM, De Rosa SC, Migueles SA, Abraham J, et al. HIV nonprogressors preferentially maintain highly functional HIV-specific CD8+ T cells. Blood. 2006;107(12):4781-9. Epub 2006/02/10. doi: 10.1182/blood-2005-12-4818. PubMed PMID: 16467198; PubMed Central PMCID: PMCPMC1895811.

19. Ferre AL, Hunt PW, Critchfield JW, Young DH, Morris MM, Garcia JC, et al. Mucosal immune responses to HIV-1 in elite controllers: a potential correlate of immune control. Blood. 2009;113(17):3978-89. Epub 2008/12/26. doi: 10.1182/blood-2008-10-182709. PubMed PMID: 19109229; PubMed Central PMCID: PMCPMC2673124.

20. Harari A, Dutoit V, Cellerai C, Bart PA, Du Pasquier RA, Pantaleo G. Functional signatures of protective antiviral T-cell immunity in human virus infections. Immunol Rev. 2006;211:236-54. Epub 2006/07/11. doi: 10.1111/j.0105-2896.2006.00395.x. PubMed PMID: 16824132 .

21. Kroner BL, Goedert JJ, Blattner WA, Wilson SE, Carrington MN, Mann DL. Concordance of human leukocyte antigen haplotype-sharing, CD4 decline and AIDS in hemophilic siblings. Multicenter Hemophilia Cohort and Hemophilia Growth and Development Studies. AIDS. 1995;9(3):275-80. Epub 1995/03/01. PubMed PMID: 7755916.

22. Fellay J, Ge D, Shianna KV, Colombo S, Ledergerber B, Cirulli ET, et al. Common genetic variation and the control of HIV-1 in humans. PLoS Genet. 2009;5(12):e1000791. Epub 2009/12/31. doi: 10.1371/journal.pgen.1000791. PubMed PMID: 20041166; PubMed Central PMCID: PMCPMC2791220.

23. International HIVCS, Pereyra F, Jia X, McLaren PJ, Telenti A, de Bakker PI, et al. The major genetic determinants of HIV-1 control affect HLA class I peptide presentation. Science. 2010;330(6010):1551-7. Epub 2010/11/06. doi: 10.1126/science.1195271. PubMed PMID: 21051598; PubMed Central PMCID: PMCPMC3235490. 
Submitted Manuscript: Confidential

540 24. Valenzuela-Ponce H, Alva-Hernandez S, Garrido-Rodriguez D, Soto-Nava M, 541 Garcia-Tellez T, Escamilla-Gomez T, et al. Novel HLA class I associations with HIV-1 542 control in a unique genetically admixed population. Sci Rep. 2018;8(1):6111. Epub 543 2018/04/19. doi: 10.1038/s41598-018-23849-7. PubMed PMID: 29666450; 544 PubMed Central PMCID: PMCPMC5904102.

545 25. Kosmrlj A, Read EL, Qi Y, Allen TM, Altfeld M, Deeks SG, et al. Effects of thymic 546 selection of the T-cell repertoire on HLA class I-associated control of HIV infection. 547 Nature. 2010;465(7296):350-4. Epub 2010/05/07. doi: 10.1038/nature08997. 548 PubMed PMID: 20445539; PubMed Central PMCID: PMCPMC3098720.

549 26. Almeida JR, Price DA, Papagno L, Arkoub ZA, Sauce D, Bornstein E, et al. 550 Superior control of HIV-1 replication by CD8+ T cells is reflected by their avidity,

551

552

553

554

555

556

557

558

559

560

561

562

563

564

565

566

567

568

569

570

571

572

573

574

575

576

577

578

579

580

581

582

583

584

585 polyfunctionality, and clonal turnover. J Exp Med. 2007;204(10):2473-85. Epub 2007/09/26. doi: 10.1084/jem.20070784. PubMed PMID: 17893201; PubMed Central PMCID: PMCPMC2118466.

27. Almeida JR, Sauce D, Price DA, Papagno L, Shin SY, Moris A, et al. Antigen sensitivity is a major determinant of CD8+ T-cell polyfunctionality and HIVsuppressive activity. Blood. 2009;113(25):6351-60. Epub 2009/04/25. doi: 10.1182/blood-2009-02-206557. PubMed PMID: 19389882; PubMed Central PMCID: PMCPMC2710928.

28. Berger CT, Frahm N, Price DA, Mothe B, Ghebremichael M, Hartman KL, et al. High-functional-avidity cytotoxic T lymphocyte responses to HLA-B-restricted Gagderived epitopes associated with relative HIV control. J Virol. 2011;85(18):9334-45. Epub 2011/07/15. doi: 10.1128/JVI.00460-11. PubMed PMID: 21752903; PubMed Central PMCID: PMCPMC3165743.

29. Mothe B, Llano A, Ibarrondo J, Zamarreno J, Schiaulini M, Miranda C, et al. CTL responses of high functional avidity and broad variant cross-reactivity are associated with HIV control. PLoS One. 2012;7(1):e29717. Epub 2012/01/13. doi: 10.1371/journal.pone.0029717. PubMed PMID: 22238642; PubMed Central PMCID: PMCPMC3251596.

30. Bailey JR, Williams TM, Siliciano RF, Blankson JN. Maintenance of viral suppression in HIV-1-infected HLA-B*57+ elite suppressors despite CTL escape mutations. J Exp Med. 2006;203(5):1357-69. Epub 2006/05/10. doi: 10.1084/jem.20052319. PubMed PMID: 16682496; PubMed Central PMCID: PMCPMC2121215.

31. Chen H, Ndhlovu ZM, Liu D, Porter LC, Fang JW, Darko S, et al. TCR clonotypes modulate the protective effect of HLA class I molecules in HIV-1 infection. Nat Immunol. 2012;13(7):691-700. Epub 2012/06/12. doi: 10.1038/ni.2342. PubMed PMID: 22683743; PubMed Central PMCID: PMCPMC3538851.

32. Draenert R, Verrill CL, Tang Y, Allen TM, Wurcel AG, Boczanowski M, et al. Persistent recognition of autologous virus by high-avidity CD8 T cells in chronic, progressive human immunodeficiency virus type 1 infection. J Virol. 2004;78(2):63041. Epub 2003/12/25. doi: 10.1128/jvi.78.2.630-641.2004. PubMed PMID: 14694094; PubMed Central PMCID: PMCPMC368768.

33. Migueles SA, Laborico AC, Imamichi H, Shupert WL, Royce C, McLaughlin M, et al. The differential ability of HLA B*5701+ long-term nonprogressors and progressors to restrict human immunodeficiency virus replication is not caused by loss of 
Submitted Manuscript: Confidential

586 recognition of autologous viral gag sequences. J Virol. 2003;77(12):6889-98. Epub 587 2003/05/28. doi: 10.1128/jvi.77.12.6889-6898.2003. PubMed PMID: 12768008; 588 PubMed Central PMCID: PMCPMC156173.

589 34. Miura T, Brumme CJ, Brockman MA, Brumme ZL, Pereyra F, Block BL, et al. 590 HLA-associated viral mutations are common in human immunodeficiency virus type 5911 elite controllers. J Virol. 2009;83(7):3407-12. Epub 2009/01/21. doi: 592 10.1128/JVI.02459-08. PubMed PMID: 19153230; PubMed Central PMCID: 593 PMCPMC2655568.

594 35. De Boer RJ. Understanding the failure of CD8+ T-cell vaccination against 595 simian/human immunodeficiency virus. J Virol. 2007;81(6):2838-48. Epub 596 2007/01/05. doi: 10.1128/JVI.01914-06. PubMed PMID: 17202215; PubMed Central 597 PMCID: PMCPMC1865966.

598 36. De Boer RJ, Homann D, Perelson AS. Different dynamics of CD4+ and CD8+ T 599 cell responses during and after acute lymphocytic choriomeningitis virus infection. J 600 Immunol. 2003;171(8):3928-35. Epub 2003/10/08. doi: 601 10.4049/jimmunol.171.8.3928. PubMed PMID: 14530309.

602 37. De Boer RJ, Perelson AS. Target cell limited and immune control models of HIV 603 infection: a comparison. J Theor Biol. 1998;190(3):201-14. Epub 1998/05/16. doi: 604 10.1006/jtbi.1997.0548. PubMed PMID: 9514649.

605 38. Wodarz D, Arnaout RA, Nowak MA, Lifson JD. Transient antiretroviral 606 treatment during acute simian immunodeficiency virus infection facilitates long-term 607 control of the virus. Philos Trans R Soc Lond B Biol Sci. 2000;355(1400):1021-9. Epub 608 2001/02/24. doi: 10.1098/rstb.2000.0639. PubMed PMID: 11186303; PubMed 609 Central PMCID: PMCPMC1692816.

610 39. Rouzine I, Coffin J. Interplay between experiment and theory in development 611 of a working model for HIV-1 population dynamics. In: Elsevier, editor. Origin and 612 Evolution of Viruses1999. p. 225-62.

613 40. Rouzine IM, Rozhnova G. Antigenic evolution of viruses in host populations. 614 PLoS Pathog. 2018;14(9):e1007291. Epub 2018/09/13. doi: 615 10.1371/journal.ppat.1007291. PubMed PMID: 30208108; PubMed Central PMCID: 616 PMCPMC6173453.

617 41. Rouzine IM, Sergeev RA, Glushtsov AI. Two types of cytotoxic lymphocyte 618 regulation explain kinetics of immune response to human immunodeficiency virus. 619 Proc Natl Acad Sci U S A. 2006;103(3):666-71. Epub 2006/01/13. doi: 620 10.1073/pnas.0510016103. PubMed PMID: 16407101; PubMed Central PMCID: 621 PMCPMC1334670.

622 42. Rouzine IM, Weinberger AD, Weinberger LS. An evolutionary role for HIV 623 latency in enhancing viral transmission. Cell. 2015;160(5):1002-12. Epub 624 2015/02/28. doi: 10.1016/j.cell.2015.02.017. PubMed PMID: 25723173; PubMed 625 Central PMCID: PMCPMC4488136.

626 43. Sergeev RA, Batorsky RE, Coffin JM, Rouzine IM. Interpreting the effect of 627 vaccination on steady state infection in animals challenged with Simian 628 immunodeficiency virus. J Theor Biol. 2010;263(3):385-92. Epub 2009/12/26. doi: 629 10.1016/j.jtbi.2009.12.018. PubMed PMID: 20034499; PubMed Central PMCID: 630 PMCPMC2827631. 
Submitted Manuscript: Confidential

631 44. Sergeev RA, Batorsky RE, Rouzine IM. Model with two types of CTL regulation 632 and experiments on CTL dynamics. J Theor Biol. 2010;263(3):369-84. Epub 633 2009/11/17. doi: 10.1016/j.jtbi.2009.11.003. PubMed PMID: 19913558; PubMed 634 Central PMCID: PMCPMC2827655.

635 45. Conway JM, Perelson AS. Post-treatment control of HIV infection. Proc Natl 636 Acad Sci U S A. 2015;112(17):5467-72. Epub 2015/04/15. doi: 637 10.1073/pnas.1419162112. PubMed PMID: 25870266; PubMed Central PMCID: 638 PMCPMC4418889.

639 46. Desikan R, Raja R, Dixit NM. Early exposure to broadly neutralizing antibodies 640 may trigger a dynamical switch from progressive disease to lasting control of SHIV 641 infection. PLoS Comput Biol. 2020;16(8):e1008064. Epub 2020/08/21. doi: 642 10.1371/journal.pcbi.1008064. PubMed PMID: 32817614; PubMed Central PMCID: 643 PMCPMC7462315.

644 47. Batorsky R, Sergeev RA, Rouzine IM. The route of HIV escape from immune 645 response targeting multiple sites is determined by the cost-benefit tradeoff of escape 646 mutations. PLoS Comput Biol. 2014;10(10):e1003878. Epub 2014/10/31. doi: 647 10.1371/journal.pcbi.1003878. PubMed PMID: 25356981; PubMed Central PMCID: 648 PMCPMC4214571.

649 48. Murphy K. Janeway's Immunobiology, Eighth Edition. London and New York: 650 Garland Science; 2011.

651 49. Zajac AJ, Blattman JN, Murali-Krishna K, Sourdive DJ, Suresh M, Altman JD, et 652 al. Viral immune evasion due to persistence of activated T cells without effector 653 function. J Exp Med. 1998;188(12):2205-13.

654 50. Biancotto A, Iglehart SJ, Vanpouille C, Condack CE, Lisco A, Ruecker E, et al. 655 HIV-1 induced activation of CD4+ T cells creates new targets for HIV-1 infection in 656 human lymphoid tissue ex vivo. Blood. 2008;111(2):699-704. Epub 2007/10/03. doi: 657 10.1182/blood-2007-05-088435. PubMed PMID: 17909079; PubMed Central PMCID: 658 PMCPMC2200839.

659 51. Coffin J, Hughes S, Varmus H, editors. Retroviruses: Cold Spring Harbor 660 Laboratory Press 2002.

661 52. Razooky BS, Pai A, Aull K, Rouzine IM, Weinberger LS. A hardwired HIV latency 662 program. Cell. 2015;160(5):990-1001. Epub 2015/02/28. doi: 663 10.1016/j.cell.2015.02.009. PubMed PMID: 25723172; PubMed Central PMCID: 664 PMCPMC4395878.

665 53. Kaufmann DE, Walker BD. PD-1 and CTLA-4 inhibitory cosignaling pathways 666 in HIV infection and the potential for therapeutic intervention. J Immunol. 667 2009;182(10):5891-7. Epub 2009/05/06. doi: 10.4049/jimmunol.0803771. PubMed 668 PMID: 19414738; PubMed Central PMCID: PMCPMC3726306.

669 54. Doitsh G, Cavrois M, Lassen KG, Zepeda O, Yang Z, Santiago ML, et al. Abortive 670

671

672

673

674

675 HIV infection mediates CD4 T cell depletion and inflammation in human lymphoid tissue. Cell. 2010;143(5):789-801. Epub 2010/11/30. doi: 10.1016/j.cell.2010.11.001. PubMed PMID: 21111238; PubMed Central PMCID: PMCPMC3026834.

55. Rong L, Perelson AS. Modeling latently infected cell activation: viral and latent reservoir persistence, and viral blips in HIV-infected patients on potent therapy. PLoS

676 Comput Biol. 2009;5(10):e1000533. Epub 2009/10/17. doi: 
Submitted Manuscript: Confidential

677 10.1371/journal.pcbi.1000533. PubMed PMID: 19834532; PubMed Central PMCID: 678 PMCPMC2752194.

679 56. Leslie A, Kavanagh D, Honeyborne I, Pfafferott K, Edwards C, Pillay T, et al. 680 Transmission and accumulation of CTL escape variants drive negative associations 681 between HIV polymorphisms and HLA. J Exp Med. 2005;201(6):891-902. Epub 682 2005/03/23. doi: 10.1084/jem.20041455. PubMed PMID: 15781581; PubMed 683 Central PMCID: PMCPMC2213090.

684 57. Friedrich TC, Dodds EJ, Yant LJ, Vojnov L, Rudersdorf R, Cullen C, et al. 685 Reversion of CTL escape-variant immunodeficiency viruses in vivo. Nat Med. 686 2004;10(3):275-81. Epub 2004/02/18. doi: 10.1038/nm998. PubMed PMID: $687 \quad 14966520$.

688 58. Troyer RM, McNevin J, Liu Y, Zhang SC, Krizan RW, Abraha A, et al. Variable 689 fitness impact of HIV-1 escape mutations to cytotoxic T lymphocyte (CTL) response. 690 PLoS Pathog. 2009;5(4):e1000365. Epub 2009/04/04. doi: 691 10.1371/journal.ppat.1000365. PubMed PMID: 19343217; PubMed Central PMCID: 692 PMCPMC2659432.

693 59. Liu Y, McNevin JP, Holte S, McElrath MJ, Mullins JI. Dynamics of viral evolution 694 and CTL responses in HIV-1 infection. PLoS One. 2011;6(1):e15639. Epub 695 2011/02/02. doi: 10.1371/journal.pone.0015639. PubMed PMID: 21283794; PubMed Central PMCID: PMCPMC3024315.

697 60. Ganusov VV, Goonetilleke N, Liu MK, Ferrari G, Shaw GM, McMichael AJ, et al. 698 Fitness costs and diversity of the cytotoxic T lymphocyte (CTL) response determine 699 the rate of CTL escape during acute and chronic phases of HIV infection. J Virol. 2011;85(20):10518-28. Epub 2011/08/13. doi: 10.1128/JVI.00655-11. PubMed PMID: 21835793; PubMed Central PMCID: PMCPMC3187476.

702 61. Khaitan A, Unutmaz D. Revisiting immune exhaustion during HIV infection.

703 Curr HIV/AIDS Rep. 2011;8(1):4-11. Epub 2010/12/29. doi: 10.1007/s11904-010-

704

705

706

707

708

709 0066-0. PubMed PMID: 21188556; PubMed Central PMCID: PMCPMC3144861.

62. Bocharov G, Klenerman P, Ehl S. Modeling the dynamics of LCMV infection in mice: II. Compartmental structure and immunopathology. J Theor Biol. 2003;221:349-78.

63. Bocharov GA. Modelling the dynamics of LCMV infection in mice: conventional and exhaustive CTL responses. J Theor Biol. 1998;192:283-308.

710 64. Rouzine I, Murali-Krishna K, Ahmed R. Generals die in friendly fire, or

711

712

713

714

715

716

717

718

719

720 modeling immune response to HIV. J Appl Comp Math. 2005;184:258-74.

65. Letvin NL, Mascola JR, Sun Y, Gorgone DA, Buzby AP, Xu L, et al. Preserved CD4+ central memory $T$ cells and survival in vaccinated SIV-challenged monkeys. Science. 2006;312(5779):1530-3. PubMed PMID: 16763152.

66. Sun Y, Schmitz JE, Buzby AP, Barker BR, Rao SS, Xu L, et al. Virus-specific cellular immune correlates of survival in vaccinated monkeys after simian immunodeficiency virus challenge. J Virol. 2006;80(22):10950-6. PubMed PMID: 16943292.

Funding: This research was funded by Agence Nationale de la Recherche, France, grant number J16R389 to I.M.R. 
Submitted Manuscript: Confidential

722 Acknowedgement: The last phase of the study was carried out within the framework

723 of the state assignment of the Federal Agency for Scientific Organizations (FASO

724 Russia: topic no. AAAA-A18-118012290142-9).

725

726 Competing interests: The funders had no role in the design of the study; in the

727 collection, analyses, or interpretation of data; in the writing of the manuscript, or in

728 the decision to publish the results.

729

730 Data and materials availability: The simulation code is available at 731 https://github.com/irouzine/HIVcontrol.

732

\section{Supplementary Material}

734

735 S1 Fig. The sensitivity of the phase diagram $\left(w, I_{0 H}\right)$ obtained from Eqs. 15,17 , and 73619 in $S 1$ Text to variation of model parameters. The default values are $c=3, I_{0 H}=$

$737 d_{H}=d_{E}=0.5, I_{0 E}=0.003$. The other parameters are as in Table 1 , unless shown 738 otherwise.

739

740 S1 Text. Mathematical appendix. 